\title{
OBESITY
}

\section{ERAD linked to obesity}

Hypothalamic neurons that secrete pro-opiomelanocortin (POMC) are known to be involved in the regulation of leptin signalling, feeding behaviour and systemic metabolic homeostasis; however, the role of the mechanisms involved in POMC prohormone folding and maturation in the endoplasmic reticulum (ER) was unknown. New research suggests that ER-associated degradation (ERAD) regulates the maturation of $\mathrm{POMC}$, which results in age-associated obesity when dysregulated.

As the SEL1L-HRD1 protein complex is a key factor in ERAD, the researchers, led by Ling Qi (University of Michigan Medical School, USA), developed a mouse model with POMC neuron-specific knock out of Sel1L (Sel1L ${ }^{\text {POMC }}$ ). These mice exhibited hyperphagia and developed obesity by 15 weeks of age, even when fed a low-energy chow diet. The researchers were also able to show that the Sel1 $L^{\text {POMC }}$ mice retained POMC in the ER, which led to hyperphagia. "We found that POMC is constantly targeted for proteasomal degradation by ERAD, and identified SEL1L-HRD1 ERAD as the mechanism linking POMC maturation to the leptin response," explains Qi. "While it is not surprising that ERAD degrades a fraction of newly synthesized unstable POMC, it is unexpected that ERAD deficiency would cause ER retention and aggregation of a large proportion of all POMC protein in vivo."

The Cys28Phe alteration in POMC is associated with early onset obesity in humans. The researchers demonstrated that Cys28Phe POMC is targeted less efficiently by ERAD for proteasomal degradation and becomes aggregated. "We showed that the maturation defects of Cys28Phe POMC can be completely rescued by an intragenic suppressor mutation at position Cys50Ser," explains Qi. The researchers are now planning to test the therapeutic potential of targeting ERAD in vivo. "We hope that more efforts will be in place in the near future to identify ERAD-targeting small molecules," concludes Qi.

\section{Claire Greenhill}

ORIGINAL ARTICLE Kim, G. H. et al.

Hypothalamic ER-associated degradation regulates POMC maturation, feeding, and ageassociated obesity. J. Clin. Invest. https://doi. org/10.1172/JCl96420(2018) 